\section{DNA and Its Epigenetic Potential, an Antenna for Cosmic Emissions: Driving Force in Evolution and Energy Transmission?}

\author{
Walter Doerfler \\ 1 Institute for Clinical and Molecular \\ Virology, Friedrich-Alexander University \\ Erlangen-Nürnberg, Erlangen, Germany
}

\section{Abstract}

Cosmic reality does not only exist but emits informative energy, completely different from cosmic radiation. Since all DNA sequences arose under the evolutionary pressure of these cosmic emissions, DNA can serve as an effective antenna to receive this unknown form of energy. Epigenetic mechanisms, known as pliable interpreter between DNA and environment, might function in capturing cosmic information transmissions. Astrophysicists have created the hypothesis of dark matter and dark energy. It is conceivable that dark energy is related to information emissions. I seek to develop an approach to detect these emissions by using DNA as bait. In case, this energy could be documented in an experimental device, enormous energy resources might open up for practical purposesadmittedly an optimistic proposal.

Keywords: Cosmic radiation; Transmission; Epigenetic; Evolution

Received: March 15, 2017; Accepted: March 20, 2017; Published: March 24, 2017
Corresponding author: Walter Doerfler

Đwalter.doerfler@t-online.de

Institute for Clinical and Molecular Virology, Friedrich-Alexander University ErlangenNürnberg Medical School, D-91054 Erlangen, Germany.

Tel: +49-9131-852-6002; +49-171-205-1587

Citation: Doerfler W. DNA and Its Epigenetic Potential, an Antenna for Cosmic Emissions: Driving Force in Evolution and Energy Transmission? J Clin Epigenet. 2017, 3:1.

\section{Modifications of the Earlier Hypothesis}

The hypothesis "DNA-a molecule in search of additional functions" postulated additional properties of deoxyribonucleic acid (DNA) [1], the carrier of all genetic information in all biological systems on this planet [2], except for the RNA viruses.

The entirety of all information (termed pool in my 2010 article), all laws governing all events in the universe, all energy and matter has not existed and does not exist in silence but emits cosmic information via an unknown physical parameter. These emissions are postulated to differ from the known forms of cosmic radiation. Obviously, we lack the sensory antennas to perceive consciously this informative energy. This transmission system may have originated simultaneously with the big bang at about 13.8 billion years ago.

These cosmic transmissions have been the evolutionary driving force for the generation of all DNA molecules with different, species-specific nucleotide sequences, the result of 4 billion years of evolution. Nucleotides are thought [3] to have been synthesized by electric discharges in a primordial earth environment. Biologically pliable and receptive molecules like oligonucleotides are capable of perceiving and interacting with these cosmic emissions whose nature we are incapable of detecting or measuring with any presently known technologies.
Over evolutionary time scales, oligonucleotides grew to the highly complex nucleotide sequences which exist today.

Of course, it is appreciated that life appears to be the rarest of rare events in the universe which originated about 9 billion years after the big bang. Hence there had to exist an extremely unlikely combination of forces to create the environment on earth to allow for the generation of DNA molecules with the ability to perceive the hypothetical cosmic transmissions.

In concert with and in addition to the formative influence from cosmic transmissions, the development of the multitude of these sequences has been affected by many different factors in the biological environments on earth which DNA has been exposed to [4]. Along these lines, highly energetic cosmic radiation has significantly determined the evolution of DNA and subsequently the origin of highly diverse forms of life on earth via random DNA mutations. However this well-known type of irradiation is different from the hypothetical form of transmissions hypothesized on in this article. For ages there has been a wide range of mutagens in the immediate environment which have contributed to conventional mutagenesis. However, in the present hypothesis consideration has been given to a more fundamental form of cosmic transmissions whose nature is completely unknown. 
Since, according to this hypothesis, DNA arose from interactions of cosmic energy transmissions, DNA is expected to be capable of "sensing" the cosmic information emissions. Possibly, DNA does exert these antenna functions only in its chromatin- or nucleusembedded conformation.

To what extent proteins could have played a role in these interactions is a matter of conjecture as well. Proteins have a more complex structure which is known to be highly adaptable to environmental conditions. To a lesser degree, DNA can also alter its conformation depending on its interactions with proteins or environmental factors.

Human DNA sequences serve as receptors and might be one of the, or the, most sophisticated one in existence. We are unaware of these transmissions since we lack the sensory reception system to respond to them. Un- or subconsciously, we might be subject to this steady flow of information transfer at all times. Quality and extent of this receptor capacity depends on the individual's DNA sequence. The DNA receptor acting as antenna might constantly export the received information to our neural system. Our psychological situation might be modulated by and psychic disorders could be related to this transfer system.

The major challenge for biology will be to devise models of how to infiltrate the system of cosmic emissions and seek to understand their character.

\section{Epigenetics-Major Antenna and Interpreter between DNA Sequence and the Environment}

Sequence-specific DNA methylations have long been known to exert a decisive role in the long-term regulation of gene activities [5]. There is conclusive evidence that the function of DNA can be modified by epigenetic mechanisms-among other factors by DNA methylation, histone modifications-in response to specific environmental factors [6-11]. Although the precise mechanisms governing these interactions between genetics and the environment are not understood, the realization that an unexpectedly variable number of environmental factors is capable of altering the methylation profiles and as a consequence the profiles of gene activities of living cells and organisms has significantly improved our understanding of genetic functions in the real world.

Of course, cosmic information transmissions might as well and preferentially seek the portal of entry, DNA, to impact the function of living cells. The patterns of DNA methylation across the entire genome are decisive in regulating genetic activities and are subject to specific alterations depending on a large number of factors from outside the genome itself. This DNA platform would be well suited to function as the receiving antenna for the emissions of cosmic information. This aspect bestows an additional important aspect on the activity modes of epigenetic mechanism dating far back in evolution.

\section{Responding to the Concepts of Dark Energy and Dark Matter}

The astrophysicists' concepts on dark energy and dark matter are about to revolutionize modern physics [12-18]. At present, ordinary (baryonic) matter which we can perceive and which has been the object of most of the work of natural scientists over centuries, including that of biologists constitutes only about $4.9 \%$ of the entirety of all matter and energy in the observable universe. Dark matter (26.8\%) and dark energy (68.3\%) have been postulated to constitute the bulk of all matter/energy. There is only indirect evidence for the existence of these ephemeral entities, and physicists today strive to explain this conundrum. As biology is a most exciting part of the universe, we are confronted with the challenge to incorporate the novel concepts of physicists into our thinking as biologists.

Among all of the natural sciences, physicists have often been at the vanguard in developing new ideas by openly questioning apparently self-evident facts and by re-examining seemingly well-established paradigms. In striving to expand their elegant edifice of today's physics, they now parse the hypothetical entities of dark energy and dark matter. Both can be deduced only from observations unexplainable by today's physics. All attempts to prove their existence directly have so far failed. Among the unresolved problems, which unsettle the thoughts of astrophysicists, are the coherence of galaxies and the accelerated expansion of the universe.

According to the classical rules of physics, the galaxies should never have been amassed. To explain the "impossibility" of the existence of billions of galaxies-including our own-a glue-like principle, the concept of dark matter has been invoked which is thought to engulf the galactic baryonic matter and keep it together. How and whether dark matter can interact with conventional matter cannot be decided at present. In a more developed stage of the dark matter hypothesis, it is suggested that dark matter is constituted by "weakly interacting massive particles", the WIMPS, which surround the baryonic matter of galaxies as an all-encompassing spherical cloud. Strongly interacting massive particles have also been considered to play a role [19] Similarly, the corollary idea of dark energy derives from the, by classical physics, unexplained observation that the universe does not only expand, but does so at steadily accelerating speed. The hitherto unknown energy responsible for this phenomenon has been postulated to be dark energy. Dark matter and dark energy should be ubiquitous and affect all events in the universe, although it has been left to our imagination how these predominant forces can interact with baryonic matter and relate to conventional forms of energy. Do we stand at the threshold to a new era in physics?

The concepts of dark matter and dark energy evoke one serious question: Are these unknown entities controlled by the known laws of physics or do they follow unknown principles, different from the laws of physics studied so far? In that case, all attempts to detect dark energy and matter with the concepts and methods of the past, might fail. As biologists, we have so far failed to 
actually take note of these revolutionary insights from physics. We continue to explore the doable with the concepts and techniques of current biology. It is time to start thinking seriously about the new physics and its impact on biology which after all is strictly governed by the laws of physics, the known and the undetected new ones alike.

\section{Implications for Biology and Medicine}

As biologists today, we are still perfecting the beautiful edifice of molecular biology and exploring the vast realms of biological and medical systems. The thought of dark matter and dark energy has not yet begun to fascinate our minds. Fields like neurobiology, evolution, interactions of the genome with the environment (epigenetic mechanisms) or unsolved problems in medicine like tumor biology, psychic disorders, and degenerative neurological ailments are in search of novel, unconventional approaches. Young investigators are not sufficiently encouraged to branch out into difficult and uncertain territories of research and to devote at least part of their intellectual energy to address these questions. And here, research institutions and academia face an inconvenient truth implicit in their present structures: The more competitive the field becomes for positions and grant support, the less affordable, because career-racking, will be attempts to engage in studies of the really unknown which will not yield immediate results. If we venture to assume DNA to play such a central role in recognizing cosmic energy of unknown characteristics, all events, all mechanisms operative in biology must be subject to these forces. We will have to join the physicists in this unusually challenging task. Physicists have accepted this challenge and started to formulate hypotheses and built impressive experimental strategies. Should the physicists be right, medicine is missing the chance to shed new light on its most pressing problems. There appear forces at work which we lack the basic means of perceiving. For today's medicine the mechanisms responsible for the causation of many complex diseases are shrouded in mystery as were infectious diseases in the pre-Louis Pasteur/Robert Koch period.

\section{Can One Exploit DNA's Evolutionary Link to Cosmic Emissions for the Exploration of their Nature and-to use DNA as Energy Antenna and Transmitter?}

This reasoning can be expanded one step further into considerations of practical applicability. I propose to exploit the possibility that DNA, chromatin or more complex sub-nuclear structures from human or plant cells, could be developed into an energy antenna and generator. As DNA is thought to have developed to its present stage in all living organisms under the pressure of cosmic information emissions, DNA might be the only biological material capable of interacting with this form of cosmic energy, of dark or other unknown, nature. The argument that dark matter and dark energy are most likely governed by a novel, hitherto completely unknown set of physical laws, reduces the chances that the new form of energy will be discovered by concepts and machinery based on the conventional laws of physics. Hence an independent approach to the discovery of dark energy has to be sought. Apart from the prospect of enabling theoreticians to unravel the nature of black energy by this approach, the possibility of opening up a completely new source of energy for practical utilization might also be of considerable interest [20].

\section{Conflict of Interest}

The author declares no conflict of interest. 


\section{References}

1 Doerfler W (2010) DNA-A molecule in search of additional functions: Recipient of pool (cosmic) wave emissions? A hypothesis. Med Hypothes 75: 291-293.

2 Avery OT, Macleod CM, McCarty M (1944) Studies on the chemical nature of the substance inducing transformation of pneumococcal types: Induction of transformation by a desoxyribonucleic acid fraction isolated from pneumo-coccus type III. J Exp Med 79: 137-158.

3 Shapiro R (1987) Origins: A skeptic's guide to the creation of life on earth. Bantam Books.

4 Darwin C (1859) On the origin of species by means of natural selection.

5 Doerfler W (1983) DNA methylation and gene activity. Annu Rev Biochem 52: 93-124.

6 Cameron NM, Shahrokh D, Del Corpo A, Dhir SK, Szyf M, et al. (2008) Epigenetic reprogramming of phenotypic variations in reproductive strategies in the rat through maternal care. J Neuroendocrinol 20 : 795-801.

7 Gershon NB, High PC (2015) Epigenetics and child abuse: Modernday Darwinsim-The miraculous ability of the human genome to adapt, and then adapt again. Am J Med Genet Semin Med Genet 169: 353-360.

8 Maccari S, Krugers HJ, Morley-Fletcher S, Szyf M, Brunton PJ (2014) The consequqnces of early life adversity: neurobiological, behavioral and epigentiv adptations. J Neuroendocrinol 26: 707-723.

9 Oh G, Petronis A (2008) Environmental studies of schizophrenia through the prism of epigenetics. Schizophr Bull 34: 1122-1129.
10 Szyf M (2013) DNA methylation, behavior and early life adversity. J Genet Genomics 40: 331-338.

11 Yet I, Tsai PC, Castillo-Fernandez JE, Carnero-Montoro E, Bell JT (2016) Genetics and environmental impacts on DNA methylation levels in twins. Epigenomics 8: 105-117.

12 Davis M, Efstathiou G, Frenk CS, White SDM (1985) The evolution of large-scale structure in a universe dominated by cold dark matter. Astrophys J 292: 371-394.

13 Perlmutter S, Aldering G, Goldhaber G, Knop RA, Nugent P, et al. (1999) Measurements of Omega and Lambda from 42 high redshift supernovae. Astrophys J 517: 565-586.

14 Riess AG, Filippenko AV, Challis P, Clocchiatti A, Diercks A, et al. (1998) Observational evidence from supernovae for an accelerating universe and a cosmological constant. Astron J 116: 1009-1038.

15 Riess AG, Livio M (2016) The puzzle of dark energy. Sci Am 314: 30-35.

16 Rubin VC, Ford W, Kent (1970) Rotation of the Andromeda Nebula from a Spectroscopic Survey of Emission Regions. Astrophys J 159: 379-403.

17 Trimble V (1987) Existence and nature of dark matter in the universe. Annu Rev Astron Astrophys 25: 425-472.

18 Vittorio N, Silk J (1984) Fine-scale anisotropy of the cosmic microwave background in a universe dominated by cold dark matter. Astrophys J Lett 285: L39-L43.

19 Merritt D, Bertone G (2005). Dark matter dynamics and indirect detection. Modern Physics Letters A 20: 1021-1036.

20 Doerfler W (2016) Beware of manipulations on the genome: epigenetic destabilization through (foreign) DNA insertions. Epigenomics 8: 587-591. 